\title{
Discrimination of the binary coherent signal: Gaussian-operation limit and simple non-Gaussian near-optimal receivers
}

\author{
Masahiro Takeoka and Masahide Sasaki \\ National Institute of Information and Communications Technology, \\ 4-2-1 Nukui-kitamachi, Koganei, Tokyo 184-8795, Japan \\ CREST, Japan Science and Technology Agency, 1-9-9 Yaesu, Chuoh-ku, Tokyo 103-0028, Japan
}

(Dated: October 23, 2018)

\begin{abstract}
We address the limit of the Gaussian operations and classical communication in the problem of quantum state discrimination. We show that the optimal Gaussian strategy for the discrimination of the binary phase shift keyed (BPSK) coherent signal is a simple homodyne detection. We also propose practical near-optimal quantum receivers that beat the BPSK homodyne limit in all areas of the signal power. Our scheme is simple and does not require realtime electrical feedback.
\end{abstract}

PACS numbers: 03.67.Hk, 42.50.Dv

\section{INTRODUCTION}

Discrimination of the binary phase shift keyed (BPSK) coherent states $\{|\alpha\rangle,|-\alpha\rangle\}$ with the minimum error is one of the most fundamental issues in optical communication and quantum signal detection theory. Coherent communication theory has been developed based on semiclassical theory where these signals are detected by homodyne measurement. For the signals with equal prior probabilities, the average error probability is given by $P_{\text {err }}=\operatorname{erfc}[\sqrt{2|\alpha|}] / 2$. This is often called the shot noise limit or the homodyne limit, falling short of the conventional error free criterion $\left(10^{-9}\right)$ when $|\alpha|^{2}<10$.

It is, however, well known that the quantum optimal receiver can largely surpass the homodyne limit. The optimal measurement is mathematically given by a twodimensional projection measurement and it attains the minimum error probability of $\left(1-\sqrt{1-e^{-4|\alpha|^{2}}}\right) / 2$ which is called the Helstrom bound [1]. Kennedy proposed a simple near-optimal receiver using a coherent local oscillator (LO) and photon counting 2]. Its error rate is only twice larger than the Helstrom bound and is smaller than the homodyne limit when $|\alpha|^{2}>0.4$. Dolinar then extended this 'Kennedy receiver' to the optimal one by introducing the adaptive electrical feedback which is enough faster than the optical signal pulse width [3] (see also 1, 4, [5, 6, 7]). Although the Dolinar's concept has been demonstrated recently [8], it is still challenging to experimentally beat the homodyne limit with this approach because of its complicated system.

From a quantum mechanical point of view, homodyne measurement belongs to the class of Gaussian operations, i.e. described by up to the second order nonlinearity, while photon counting is the non-Gaussian one. The role of Gaussian operations in quantum information protocols [9] or quantum state estimation [10] have been widely investigated. On the other hand, it has also been shown that some of the important protocols cannot be performed by only Gaussian operations and classical communication (GOCC) and inevitably requires non-Gaussian operations, e.g. quantum computing [1]], entanglement distillation of Gaussian states [12, 13, 14], and the optimal cloning of coherent states [15].

In this paper, we first show that the homodyne measurement is the best strategy to discriminate the binary coherent states within GOCC. To our knowledge, this is the first result addressing the Gaussian limit in quantum state discrimination scenario. In the second half of the paper, we propose novel non-Gaussian quantum receivers based on the Kennedy receiver, that beat the Gaussian limit for any $|\alpha|^{2}$. In particular, we point out that the amount of displacement in the Kennedy receiver is not optimal. Our schemes do not require realtime feedback and are simple and practical to experimentally overcome the homodyne limit with current technology.

\section{DISCRIMINATION VIA GAUSSIAN OPERATIONS AND CONDITIONAL DYNAMICS}

In this section, we show that "the minimum error discrimination of a set of two coherent states $\{|\alpha\rangle,|-\alpha\rangle\}$ with the prior probabilities $\left\{p_{+}, p_{-}\right\}$under GOCC is attained by the homodyne detection". For simplicity, $\alpha$ is assumed to be real. Gaussian operation is defined as the operation that maps Gaussian states to Gaussian states. For Gaussian input states, properties of Gaussian operations have been well investigated [13, 14]. In our problem, however, although each signal state is given by a Gaussian state, the signal from the receiver's viewpoint is an ensemble of these states, $\hat{\rho}_{i}=p_{+}|\alpha\rangle\left\langle\alpha\left|+p_{-}\right|-\alpha\right\rangle\langle-\alpha|$, i.e. non-Gaussian. This is because the receiver does not know which state he or she is receiving. We therefore start by revisiting the measurement processes based on GOCC.

\section{A. Characterization of the measurements with GOCC}

It is known that any Gaussian operation (Gaussian completely positive (CP) map) in optical system can be implemented by adding an ancillary system prepared in 
Gaussian state, applying Gaussian unitary operation on the whole system (implementable via linear optics and squeezing), and then discarding and/or performing homodyne measurements on a part of the system [14]. The CP map including measurements is not always tracepreserving and the output quantum state might be conditioned on the measurement outcome. When Gaussian operations are sequentially applied, the measurement outcomes (classical information) are sometimes useful to dynamically renew each step of quantum operations, which is called conditional dynamics. In the following, we characterize two types of measurements consisting of Gaussian operation with and without conditional dynamics.

The first one is the measurement with only Gaussian quantum operation (without conditional dynamics). Here we call it a 'Gaussian measurement'. A generic physical model of the Gaussian measurement is depicted in Fig. 1(a), consisting of Gaussian unitary operation, Gaussian ancillary states, and homodyne detectors. After the Gaussian measurement is finished, a set of measurement outcome is classically post-processed, in our case, to make the decision which signal is detected. Throughout this section, we consider only 'noise-free' operations and measurements, that is we assume that ancillary states are always pure and the system is never discarded. It does not lose generality. Since to discard some of the modes means to lose their information, it is realized by measuring them via homodyne detectors and ignoring the outcomes, where the latter is included in classical post-processing. Mixed ancillary states are provided by first preparing entangled pure states and then discarding a part of them, therefore, can be prepared by pure states and post-processing. These imply the generality of the noise-free model. A Gaussian measurement detecting an $L$-mode quantum state is mathematically described by a positive operator-valued measure (POVM) $\left\{\hat{\Pi}_{\mathrm{G}}(\Gamma, \delta)\right\}_{\delta}$ where $\hat{\Pi}_{\mathrm{G}}(\Gamma, \delta)>0, \int d \delta \hat{\Pi}_{\mathrm{G}}(\Gamma, \delta)=\hat{I}$, and $\hat{I}$ is an identity operator (see Appendix for its derivation from the physical model). The operator $\hat{\Pi}_{G}(\Gamma, \delta)$ is a Gaussian operator, i.e. its characteristic function is described by $\chi(\omega)=\exp \left[-\frac{1}{4} \omega^{T} \Gamma \omega+i \delta^{T} \omega\right]$ where $\Gamma$ and $\delta$ are the $2 L \times 2 L$ covariance matrix and the $2 L$-dimensional displacement vector, respectively, and $T$ is the transpose operation.

The second one consists of GOCC which we call a 'GOCC-measurement'. As illustrated in Fig. 1(b), its generic model is described by sequential Gaussian operations with conditional dynamics via classical communication. Each step of Gaussian operation includes Gaussian operation and ancillary states, and a Gaussian measurement detecting a part of the system. The measurement outcomes are applied to modify the following step of Gaussian operations in realtime, which is the conditional dynamics via classical communication. After the whole quantum measurement process is finished, all of the measurement outcomes is used for the classical postprocessing. The whole process except the post-processing is described by a POVM $\left\{\hat{\Pi}_{\mathrm{GOCC}}(\Gamma(x), \delta(x))\right\}_{x}$ with a

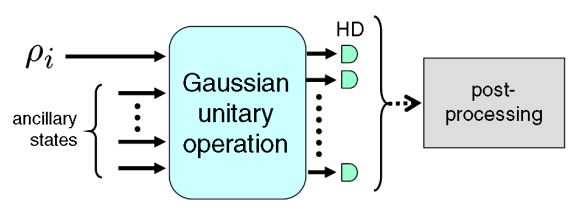

(a)

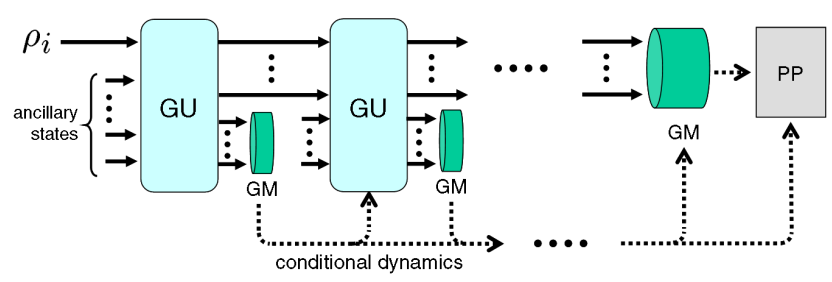

(b)

FIG. 1: (Color online) Generic physical models of (a) Gaussian measurement and (b) GOCC-measurement. The solid and dotted lines represent quantum and classical signals, respectively. HD: homodyne detector, GU: Gaussian unitary operation, GM: Gaussian measurement, PP: post-processing.

covariance matrix $\Gamma(x)$ and a displacement $\delta(x)$ where $\hat{\Pi}_{\mathrm{GOCC}}(\Gamma(x), \delta(x))>0$ and $\int d x \hat{\Pi}_{\mathrm{GOCC}}(\Gamma(x), \delta(x))=\hat{I}$. The parameter $x$ indicates the pattern of which conditional dynamics is applied during the whole process. Again, throughout the section, we restrict the GOCCmeasurements to be noise-free.

Gaussian measurement is well characterized and easily applied to the optimization problem on the state discrimination. On the other hand, although GOCCmeasurement is also well defined, it is not easy to handle its POVM directly. To prove the optimality of the homodyne limit under GOCC-measurements, therefore, we first show that the optimal Gaussian measurement without CC is a homodyne measurement. Then we discuss an important property of the conditional output from a Gaussian operation with an input of binary coherent state signals. Finally, we prove that even in the GOCCmeasurement scenario, conditional dynamics is not useful and thus a simple homodyne measurement is optimal.

\section{B. Optimal Gaussian measurement}

A Gaussian measurement for the single-mode input state is described by $\left\{\hat{\Pi}\left(\Gamma_{\mathcal{M}}, D_{\mathcal{M}}\right)\right\}_{D_{\mathcal{M}}}$ where $D_{\mathcal{M}}$ is a two-dimensional vector and

$$
\Gamma_{\mathcal{M}}=\left[\begin{array}{cc}
c_{-} & s \\
s & c_{+}
\end{array}\right]
$$

where $c_{ \pm}=\cosh (2 r) \pm \sinh (2 r) \cos \varphi, s=\sinh (2 r) \sin \varphi$, and $r$ and $\varphi$ are the real parameters. The minimum error probability to discriminate $\left\{| \pm \alpha\rangle, p_{ \pm}\right\}$by $\left\{\hat{\Pi}\left(\Gamma_{\mathcal{M}}, D_{\mathcal{M}}\right)\right\}_{D_{\mathcal{M}}}$ with given $r$ and $\varphi$ can be calculated from the probability distribution to detect each signal 
$P_{ \pm}\left(D_{\mathcal{M}}\right)=\left\langle \pm \alpha\left|\hat{\Pi}\left(\Gamma_{\mathcal{M}}, D_{\mathcal{M}}\right)\right| \pm \alpha\right\rangle$. Applying a conventional Bayesian decision strategy as a post-processing, we obtain

$$
\begin{aligned}
P_{e}^{(G)}= & \frac{p_{+}}{2} \operatorname{erfc}\left[e \sqrt{2} \alpha+\frac{\ln \left(p_{+} / p_{-}\right)}{4 e \sqrt{2} \alpha}\right] \\
& +\frac{p_{-}}{2} \operatorname{erfc}\left[e \sqrt{2} \alpha-\frac{\ln \left(p_{+} / p_{-}\right)}{4 e \sqrt{2} \alpha}\right],
\end{aligned}
$$

where

$$
e=\frac{1+\cosh (2 r)+\sinh (2 r) \cos \varphi}{2(1+\cosh (2 r))} .
$$

It is apparent that $P_{e}^{(G)}$ is minimum when $\varphi=0$ and $r=\infty$, which implies that the homodyne detection with the phase $\varphi=0$ is the optimal strategy within all possible Gaussian measurements.

\section{Conditional output states from a Gaussian operation}

As mentioned above, a GOCC-measurement consists of a sequence of Gaussian operations that include partial measurements. In this subsection, before discussing a whole GOCC-measurement process, we pick up one step of the sequence and address a useful property of the conditional output from a Gaussian operation with the binary coherent state inputs.

Let us consider the noise-free conditional Gaussian operation which transforms a single-mode input to an $N$-mode output where the output state is conditioned on a partial measurement outcome $d_{\mathcal{M}}$. Suppose an input state is $|\alpha\rangle$ or $|-\alpha\rangle$. We show that for any $d_{\mathcal{M}}$, the conditional output states of the inputs $| \pm \alpha\rangle$ can always be transformed to $\left|\alpha_{ \pm}^{\prime}\right\rangle\left\langle\alpha_{ \pm}^{\prime}\right| \otimes \hat{\rho}_{\text {aux }}^{\prime}$ by the same $d_{\mathcal{M}}$-independent deterministic Gaussian operation, where $\alpha_{ \pm}^{\prime}= \pm \alpha^{\prime}+\bar{\alpha}^{\prime}\left(d_{\mathcal{M}}\right)$ and $\alpha^{\prime}$ is independent of $d_{\mathcal{M}}$. We also show that, for a statistical ensemble input $\hat{\rho}_{i}=p_{+}|\alpha\rangle\left\langle\alpha\left|+p_{-}\right|-\alpha\right\rangle\langle-\alpha|$, the conditional output is similarly transformed to be $\hat{\rho}_{i}^{\prime} \otimes \hat{\rho}_{\text {aux }}^{\prime}$ where

$$
\hat{\rho}_{i}^{\prime}=p_{+}^{\prime}\left(d_{\mathcal{M}}\right)\left|\alpha_{+}^{\prime}\right\rangle\left\langle\alpha_{+}^{\prime}\left|+p_{-}^{\prime}\left(d_{\mathcal{M}}\right)\right| \alpha_{-}^{\prime}\right\rangle\left\langle\alpha_{-}^{\prime}\right| .
$$

Let $\hat{\rho}(\gamma, d)$ be a density matrix of a Gaussian state with a covariance matrix $\gamma$ and a displacement $d$. For example, the coherent states $| \pm \alpha\rangle$ are denoted as $\hat{\rho}\left(I_{2}, \pm d_{\alpha}\right)$ where $\pm d_{\alpha}=[ \pm \sqrt{2} \alpha, 0]^{T}$ and $I_{2 L}$ is a $2 L \times 2 L$ identity matrix. The conditional operation is described as follows. The initial single-mode state is interacted with $M-1$ Gaussian auxiliary states $(M>N)$ via a Gaussian unitary operation. Without loss of generality, we can set the auxiliary states to be $M-1$ vacua. At the covariance matrix level, Gaussian unitary operation is described by the matrix transformation via a symplectic matrix $S$ and an additional displacement $\bar{d}$. These transform the coher- ent states $\hat{\rho}\left(I_{2}, \pm d_{\alpha}\right)$ as

$$
\begin{aligned}
I_{2} & \rightarrow S I_{2} \oplus I_{2(M-1)} S^{T} \equiv \gamma, \\
\pm d_{\alpha} & \rightarrow S[ \pm \sqrt{2} \alpha, 0, \cdots, 0]^{T}+\bar{d} \equiv \pm d+\bar{d},
\end{aligned}
$$

where $S$ and $\gamma$ are $2 M \times 2 M$ matrices and $d$ and $\bar{d}$ are $2 M$-dimensional vectors. The $N$-mode conditional output is obtained by performing an $(M-N)$-mode noisefree Gaussian measurement $\left\{\hat{\Pi}\left(\gamma_{\mathcal{M}}, d_{\mathcal{M}}\right)\right\}_{d_{\mathcal{M}}}$. For convenience, we divide the system by the first $N$ modes and the remaining $M-N$ modes and call them the system $\mathrm{A}$ and $\mathrm{B}$, respectively, as

$$
\gamma=\left[\begin{array}{cc}
A & C \\
C^{T} & B
\end{array}\right], \quad d=\left[\begin{array}{l}
d^{A} \\
d^{B}
\end{array}\right], \quad \bar{d}=\left[\begin{array}{l}
\bar{d}^{A} \\
\bar{d}^{B}
\end{array}\right] .
$$

After performing the Gaussian measurement on the system $B$, each of the signals $(| \pm \alpha\rangle)$ is transformed to an $N$-mode conditional output state as

$$
\hat{\rho}\left(I_{2}, \pm d_{\alpha}\right) \rightarrow \hat{\rho}\left(\Gamma_{\text {out }}, D_{ \pm}\right)
$$

where [14]

$$
\begin{aligned}
\Gamma_{\text {out }}= & A-C \frac{1}{B+\gamma_{\mathcal{M}}} C^{T}, \\
D_{ \pm}= & \pm\left(d^{A}-C \frac{1}{B+\gamma_{\mathcal{M}}} d^{B}\right) \\
& +\bar{d}^{A}-C \frac{1}{B+\gamma_{\mathcal{M}}}\left(\bar{d}^{B}-d_{\mathcal{M}}\right) \\
\equiv & \pm D+\bar{D}_{\mathcal{M}} .
\end{aligned}
$$

Note that each of $\hat{\rho}\left(\Gamma_{\text {out }}, D_{ \pm}\right)$is a pure state since the operations are noise-free.

Let us show that $\hat{\rho}\left(\Gamma_{\text {out }}, D_{ \pm}\right)$can be simultaneously transformed to $\left|\alpha_{ \pm}^{\prime}\right\rangle\left\langle\alpha_{ \pm}^{\prime}\right| \otimes \hat{\rho}_{\text {aux }}^{\prime}$ via Gaussian unitary operations. Since each of $\hat{\rho}\left(\Gamma_{\text {out }}, D_{ \pm}\right)$is a pure state, there exists a symplectic transformation (i.e. Gaussian unitary operation) $S_{D}$ such that [17]

$$
\Gamma_{\text {out }} \rightarrow S_{D} \Gamma_{\text {out }} S_{D}^{T}=I_{2 N},
$$

where the displacement is also transformed as

$$
D_{ \pm} \rightarrow \pm S_{D} D+S_{D} \bar{D}_{\mathcal{M}}
$$

Note that $S_{D}$ depends only on $\Gamma_{\text {out }}$ and thus independent of $d_{\mathcal{M}}$. Let $S_{D} D \equiv\left[d_{1}, d_{2}, \cdots, d_{2 N}\right]^{T}$ and $S_{D} \bar{D}_{\mathcal{M}} \equiv\left[\bar{d}_{1}, \bar{d}_{2}, \cdots, \bar{d}_{2 N}\right]^{T}$. We can transform them to $\left[ \pm d^{\prime}, 0, \cdots, 0\right]^{T}$ and $\left[\bar{d}_{1}^{\prime}, \bar{d}_{2}^{\prime}, \cdots, \bar{d}_{2 N}^{\prime}\right]^{T}$, respectively, by some combination of linear optics (beamsplitters and phase shifters) where the covariance matrix $I_{2 N}$ is kept to be invariant. Again parameters of the beamsplitters depend only on $\left\{d_{i}\right\}_{i}$, and independent of $\left\{\bar{d}_{i}\right\}_{i}$, i.e. free from $d_{\mathcal{M}}$. After these operations, the states are transformed to be the desired ones

$$
\hat{\rho}\left(\Gamma_{\text {out }}, D_{ \pm}\right) \rightarrow \hat{\rho}\left(I_{2},\left[ \pm d^{\prime}+\bar{d}_{1}^{\prime}, \bar{d}_{2}^{\prime}\right]^{T}\right) \otimes \hat{\rho}_{\text {aux }}^{\prime}
$$


where $\hat{\rho}_{\text {aux }}^{\prime}$ is a product of $N-1$ coherent states with the displacement $\left[\bar{d}_{3}^{\prime}, \bar{d}_{4}^{\prime}, \cdots, \bar{d}_{2 N}^{\prime}\right]^{T}$. These are the desired ones.

Finally we apply the above scenario onto the initial state of $\hat{\rho}_{i}=p_{+} \hat{\rho}\left(I_{2},+d_{\alpha}\right)+p_{-} \hat{\rho}\left(I_{2},-d_{\alpha}\right)$. Following the above procedures, its conditional output after the Gaussian operation is given by

$\hat{\rho}_{\text {out }}=p_{+} P_{+}\left(d_{\mathcal{M}}\right) \hat{\rho}\left(\Gamma_{\text {out }}, D_{+}\right)+p_{-} P_{-}\left(d_{\mathcal{M}}\right) \hat{\rho}\left(\Gamma_{\text {out }}, D_{-}\right)$,

where

$$
\begin{aligned}
P_{ \pm}\left(d_{\mathcal{M}}\right)= & \frac{1}{\sqrt{\operatorname{det}\left(B+\gamma_{\mathcal{M}}\right)}} \exp \left[-\left( \pm d^{B}+\bar{d}^{B}-d_{\mathcal{M}}\right)^{T}\right. \\
& \left.\times \frac{1}{B+\gamma_{\mathcal{M}}}\left( \pm d^{B}+\bar{d}^{B}-d_{\mathcal{M}}\right)\right]
\end{aligned}
$$

After the unitary operation of $S_{D}$ and appropriate linear operations, the state is transformed to be

$$
\hat{\rho}_{\text {out }} \rightarrow \hat{\rho}_{i}^{\prime} \otimes \hat{\rho}_{\text {aux }}^{\prime}
$$

where

$$
\begin{aligned}
\hat{\rho}_{i}^{\prime}= & p_{+} P_{+}\left(d_{\mathcal{M}}\right) \hat{\rho}\left(I_{2},\left[d^{\prime}+\bar{d}_{1}^{\prime}, \bar{d}_{2}^{\prime}\right]^{T}\right) \\
& +p_{-} P_{-}\left(d_{\mathcal{M}}\right) \hat{\rho}\left(I_{2},\left[-d^{\prime}+\bar{d}_{1}^{\prime}, \bar{d}_{2}^{\prime}\right]^{T}\right) .
\end{aligned}
$$

\section{Optimal GOCC-measurement}

Let us now turn to the state discrimination via GOCCmeasurements. To specify the role of conditional dynamics, we first consider a simpler measurement scenario where a single conditional Gaussian operation and a Gaussian measurement are sequentially operated on the signal. Denote the partial measurement outcome at the former step as $d_{\mathcal{M}}$, which is informed to the latter measurement step to optimize the process of Gaussian measurement. After these GOCC processes, all measurement outcomes are classically post-processed.

Applying the result in the previous subsection to the initial state of $\hat{\rho}_{i}$, the conditional output from the first Gaussian operation can be transformed to corresponding $\hat{\rho}_{i}^{\prime}$ described in Eq. (17) via $d_{\mathcal{M}}$-independent deterministic Gaussian operations. Let this operation be a part of the second step Gaussian measurement (if necesssary, one can add $\hat{\rho}_{\text {aux }}^{\prime}$ as an ancilla). Then the remaining task in the measurement is to discriminate two coherent states $\left\{\left|\alpha_{+}^{\prime}\right\rangle,\left|\alpha_{-}^{\prime}\right\rangle\right\}$ with the prior probabilities of $\left\{p_{+}^{\prime}\left(d_{\mathcal{M}}\right), p_{-}^{\prime}\left(d_{\mathcal{M}}\right)\right\}$. As already mentioned, the optimal Gaussian measurement is given by a simple homodyne detection. Its phase $\varphi$ is determined by geometric configuration between $\alpha_{+}^{\prime}$ and $\alpha_{-}^{\prime}$ and since $\alpha_{ \pm}^{\prime}=\left( \pm d^{\prime}+\bar{d}_{1}^{\prime}+i \bar{d}_{2}^{\prime}\right) / \sqrt{2}$ (see Eq. (17)), it is always given by $\varphi=0$ which is irrespective to the values of $\bar{d}_{1}^{\prime}$, $\bar{d}_{2}^{\prime}$ and thus also $d_{\mathcal{M}}$. An optimal strategy for the second step Gaussian measurement therefore consists of the

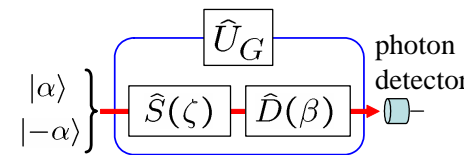

(a)

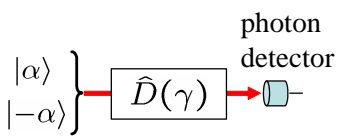

(b)
FIG. 2: (Color online) Schematic of the near-optimal quantum receivers. (a) Type-I: Photon detector + optimal Gaussian unitary operation. (b) Type-II: Photon detector + optimal displacement.

transformation $\hat{\rho}_{\text {out }} \rightarrow \hat{\rho}_{i}^{\prime}$ and the homodyne detection where any parameters in those processes are independent of $d_{\mathcal{M}}$. It implies that the conditional dynamics is not necessary for to optimize the second step measurement [18]. Consequently, the optimal whole process of these steps is described by a Gaussian measurement and thus, as already shown, is a homodyne measurement. Note that this statement is obtainable without specifying a concrete process of the first step Gaussian operation. An extension of the above scenario to the multi-step one is straightforward, which proves the optimality of the homodyne measurement within all possible GOCC-measurement.

\section{NEAR-OPTIMAL QUANTUM RECEIVER BY USING A PHOTON COUNTER}

The homodyne limit (GOCC limit) stated in the previous section is overcome by adding a non-Gaussian measurement device. In this section, by extending the Kennedy receiver, we propose a simple near-optimal receiver where a photon counter, which is a typical nonGaussian operation device, is added to the prior Gaussian operation. In what follows, we assume $p_{+}=p_{-}=1 / 2$ for simplicity.

In the Kennedy receiver, the BPSK signal $\{|\alpha\rangle,|-\alpha\rangle\}$ is shifted to $\{|2 \alpha\rangle,|0\rangle\}$ by the displacement operation $\hat{D}(\alpha)=\exp \left(\alpha \hat{a}^{\dagger}-\alpha^{*} \hat{a}\right)$, where $\hat{a}$ and $\hat{a}^{\dagger}$ are annihilation and creation operators, respectively, and then detected by an on/off type photon detector which discriminates zero or non-zero photons. It is well known that $\hat{D}(\alpha)$ can be realized by using a beamsplitter with the transmittance $\tau \rightarrow 1$ and the coherent LO $|\alpha / \sqrt{\tau}\rangle$. Here, we extend the Kennedy receiver and consider the setup depicted in Fig. 2(a), where the displacement $\hat{D}(\alpha)$ is replaced by a single-mode general Gaussian unitary operation $\hat{U}_{G}$. We will seek its optimal structure.

An on/off detector is described by the POVM $\left\{\hat{\Pi}_{\text {off }}, \hat{\Pi}_{\text {on }}\right\}$ with

$$
\hat{\Pi}_{\mathrm{off}}=e^{-\nu} \sum_{m=0}^{\infty}(1-\eta)^{m}|m\rangle\langle m|, \quad \hat{\Pi}_{\mathrm{on}}=\hat{I}-\hat{\Pi}_{\mathrm{off}}
$$

where $|m\rangle$ is an $m$-photon state, $\eta$ is the quantum effi- 
ciency, and $\nu$ is the dark counts. The Gaussian unitary operation $\hat{U}_{G}$ consists of phase shift, displacement, and squeezing, while one can omit the phase shift since the on/off detection is insensitive to the global phase. Then the average error probability is calculated from

$$
P_{e}=\frac{1}{2}\left(\left\langle\alpha\left|\hat{U}_{G}^{\dagger} \hat{\Pi}_{\mathrm{off}} \hat{U}_{G}\right| \alpha\right\rangle+\left\langle-\alpha\left|\hat{U}_{G}^{\dagger} \hat{\Pi}_{\mathrm{on}} \hat{U}_{G}\right|-\alpha\right\rangle\right),
$$

where $\hat{U}_{G}=\hat{D}(\beta) \hat{S}(\zeta), \hat{S}(\zeta)=\exp \left[\frac{1}{2}\left(\zeta^{*} \hat{a}^{2}-\zeta \hat{a}^{\dagger}\right)\right]$ is the squeezing operator, and $\zeta=r e^{i \varphi}$ is the complex squeezing parameter.

After some algebra, one can find that $r, \varphi$, and $\beta$ have the extreme at the same point, where $\varphi=0, \beta$ is real, and the optimal displacement $\beta_{\mathrm{opt}}$ and squeezing $r_{\mathrm{opt}}$, are given by $\beta$ and $r$ satisfying

$$
\begin{aligned}
\frac{8 \eta \alpha \beta}{1-e^{4 r}}= & \left\{\frac{4 \eta\left(\alpha^{2}+\beta^{2}\right)}{1-e^{4 r}}-\frac{\eta+(2-\eta) e^{-2 r}}{\eta+(2-\eta) e^{2 r}}\right\} \\
& \times \tanh \left(\frac{4 \eta \alpha \beta}{\eta+(2-\eta) e^{-2 r}}\right), \\
\alpha= & \beta \tanh \left(\frac{4 \eta \alpha \beta}{\eta+(2-\eta) e^{-2 r}}\right),
\end{aligned}
$$

simultaneously. The optimized average error probability is then given by

$$
\begin{aligned}
P_{e}^{D S}= & \frac{1}{2}-\frac{2 e^{-\nu}}{\sqrt{\left(\eta+(2-\eta) e^{2 r_{\mathrm{opt}}}\right)\left(\eta+(2-\eta) e^{-2 r_{\mathrm{opt}}}\right)}} \\
& \times \exp \left[-\frac{2 \eta\left(\alpha^{2}+\beta_{\mathrm{opt}}^{2}\right)}{\eta+(2-\eta) e^{-2 r_{\mathrm{opt}}}}\right] \\
& \times \sinh \left[\frac{4 \eta \alpha \beta_{\mathrm{opt}}}{\eta+(2-\eta) e^{-2 r_{\mathrm{opt}}}}\right] .
\end{aligned}
$$

In the following, we call it as the Type-I receiver. It should be noted that if one can use an arbitrarily higher order nonlinear unitary operation instead of $\hat{U}_{G}$, it is able to achieve the Helstrom bound rigorously [4].

On the other hand, if one is restricted to use only linear unitary operation, that is the displacement $\hat{D}(\gamma)$, the conditions in Eqs. (20) and (21) are simplified as

$$
\alpha=\gamma \tanh (2 \eta \alpha \gamma)
$$

The schematic is shown in Fig. 2(b) and we call it the Type-II receiver. Its average error probability is given by

$$
P_{e}^{D}=\frac{1}{2}-e^{-\nu-\eta\left(\alpha^{2}+\gamma_{\mathrm{opt}}^{2}\right)} \sinh \left(2 \eta \alpha \gamma_{\mathrm{opt}}\right),
$$

where $\gamma_{\text {opt }}$ is the $\gamma$ satisfying Eq. (23). Note that its physical setup is the same as that of the Kennedy receiver. However, we stress that $\gamma_{\text {opt }} \neq \alpha$ in general and thus the conventional Kennedy receiver is easily improved by using $\hat{D}\left(\gamma_{\text {opt }}\right)$ instead of $\hat{D}(\alpha)$. Figure 3(a) plots the average error probabilities for the Type-I, TypeII, and Kennedy receivers, the homodyne limit, and the Helstrom bound while $r_{\mathrm{opt}}, \beta_{\mathrm{opt}}$, and $\gamma_{\mathrm{opt}}$ are shown in
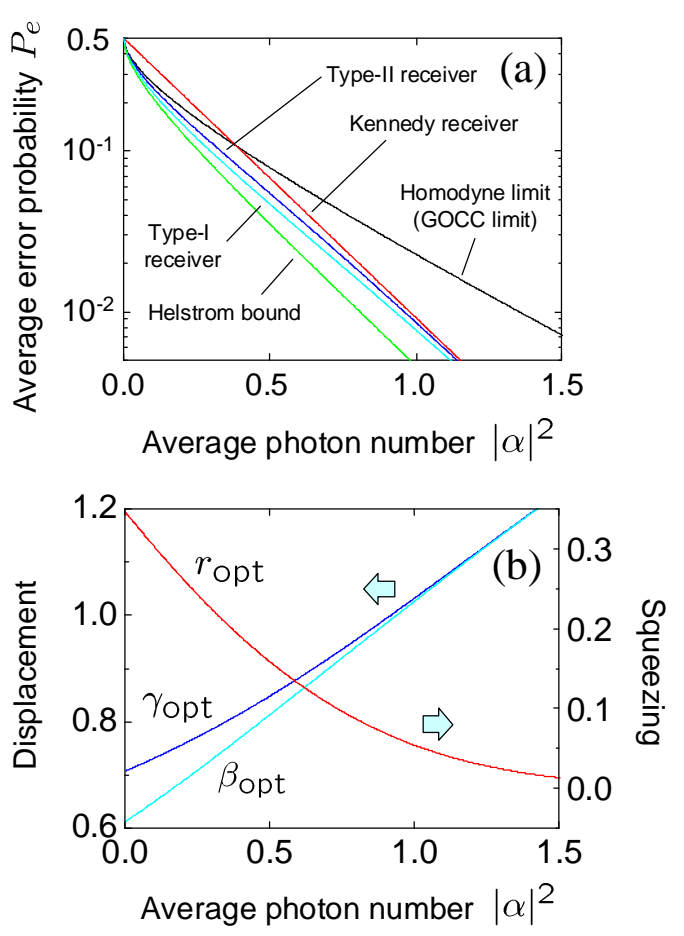

FIG. 3: (Color online) (a) Average error probabilities for the Type-I, Type-II, and Kennedy receivers, the homodyne limit, and the Helstrom bound. (b) The optimal displacements and squeezing for the Type-I and II receivers.

Fig. 3(b). It is shown that the error probabilities for both the Type-I and II receivers are better than the homodyne limit for any $|\alpha|^{2}$.

Let us finally discuss the practical perspective of these non-Gaussian receivers, particularly, the Type-II receiver. The superiority of the Type-II receiver rather than the Kennedy receiver in $|\alpha|^{2} \leq 1$ is significant to beat the homodyne limit in realistic experiments. It is known that the Kennedy-type receiver is not robust against thermal noise or dark counts [19]. Moreover, even without environmental noises, the mode mismatch between the signal and LO causes additional dark counts. As mentioned above, the displacement $\hat{D}(\beta)$ is realized by interfering the signal with the coherent state LO $|\beta / \sqrt{1-\tau}\rangle$ via the beamsplitter of the transmittance $\tau$. The effect of mode mismatch can be characterized by introducing the mode match factor $\xi(0 \leq \xi \leq 1)$ representing the overlap between the signal and LO pulse areas. Since these two pulses are in a coherent state, the average intensity of the signal field after the interference is simply given by

$$
I=(1-\xi)\left(\tau|\alpha|^{2}+|\beta|^{2}\right)+\xi| \pm \sqrt{\tau} \alpha+\beta|^{2}
$$

Due to its Poissonian photon number distribution, the average discrimination error including $\tau$ and $\xi$ at the 


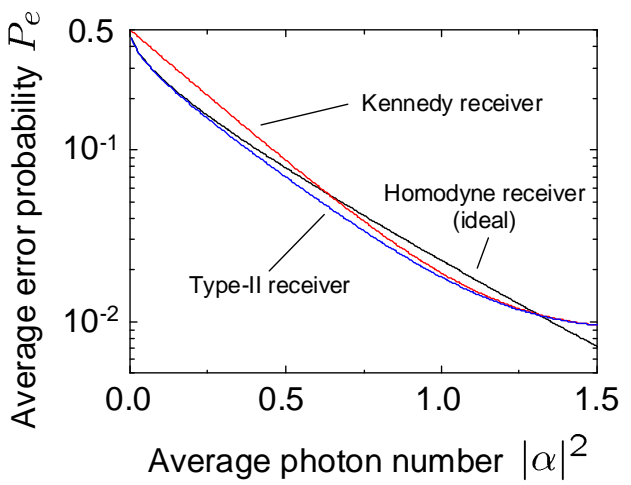

FIG. 4: (Color online) Average error probabilities for the ideal homodyne receiver and the Type-II and Kennedy receivers with practical imperfections, $\tau=0.99, \eta=0.9, \nu=10^{-3}$, and $\xi=0.995$.

on/off detector is described as

$$
\tilde{P}_{e}^{D}=\frac{1}{2}-e^{-\nu-\eta\left(\tau \alpha^{2}+\tilde{\gamma}_{\mathrm{opt}}^{2}\right)} \sinh \left(2 \eta \xi \sqrt{\tau} \alpha \tilde{\gamma}_{\mathrm{opt}}\right),
$$

where $\tilde{\gamma}_{\text {opt }}$ fulfills the optimality condition

$$
\xi \sqrt{\tau} \alpha=\tilde{\gamma}_{\text {opt }} \tanh \left(2 \eta \xi \alpha \tilde{\gamma}_{\text {opt }}\right) .
$$

An example of the average error probabilities including the imperfections is shown in Fig. 4 which clearly shows the advantage of our proposed receiver would be crucial to experimentally observe the gain of the non-Gaussian measurement beyond the homodyne limit. Although the requirement for $\eta$ in the weaker signal is still high, recent experimental progress in this field is rather promising [21].

\section{CONCLUSIONS}

In this paper, we have addressed the discrimination of the BPSK signals and proved that the homodyne limit is the minimum error probability attainable via Gaussian operations and classical communication. This is the first clarification of the limit of Gaussian operation in the state discrimination problem. Although it is shown for the binary coherent states that any conditional dynamics due to $\mathrm{CC}$ is not effective, we note that this would not be the case for the discrimination of more than two signals. Related to this topic, an increase of the mutual information by the adaptive homodyne strategies has been numerically observed [20]. For further investigation into this direction, more useful formulation of the GOCC-measurement would be necessary.

We have also proposed the near-optimal quantum receivers for the BPSK coherent signals, that are based on a photon detector and Gaussian operations. Our schemes are simple and do not require realtime electrical feedback although their error probabilities are better than the homodyne limit for any signal photon number region. Because of the recent experimental progress of high efficiency photon detectors [21] and universal squeezing operations [22], we believe that now it would be feasible in near future to beat the homodyne limit in digital optical communication experiments.

Note added: Proof-of-principle experiment of the Type-II receiver is recently demonstrated [23].

\section{Acknowledgments}

We would like to thank stimulating and encouraging discussions with U. L. Andersen, M. Ban, R. Filip, L. Mista, and C. Wittmann. We also acknowledge valuable comments from the referee. This work was supported by a MEXT Grant-in-Aid for Young Scientists (B) 19740253.

\section{APPENDIX: GENERIC MODEL OF THE GPOVM}

Here we show that the POVM of the physical model illustrated in Fig. 1(a) is always described by $\left\{\hat{\Pi}_{G}(\Gamma, \delta)\right\}_{\delta}$ introduced in Sec. IIA. Let $\hat{\rho}_{\text {sig }}^{A}$ and $\hat{\rho}_{\text {aux }}^{B}$ be an $N_{A}$-mode input state and an $N_{B}$-mode ancillary state, respectively. The probability distribution of the measurement is given by

$$
\begin{aligned}
p\left(d_{\mathrm{HD}}\right) & =\operatorname{Tr}_{A B}\left[\left(\hat{U}_{S}^{A B} \hat{\rho}_{\mathrm{sig}}^{A} \otimes \hat{\rho}_{\mathrm{aux}}^{B} \hat{U}_{S}^{A B \dagger}\right) \hat{\Pi}_{\mathrm{HD}}^{A B}\left(\Gamma_{\mathrm{HD}}, d_{\mathrm{HD}}\right)\right] \\
& =\operatorname{Tr}_{A}\left[\hat{\rho}_{\mathrm{sig}}^{A} \operatorname{Tr}_{B}\left[\hat{\rho}_{\mathrm{aux}}^{B} \hat{U}_{S}^{A B \dagger} \hat{\Pi}_{\mathrm{HD}}^{A B}\left(\Gamma_{\mathrm{HD}}, d_{\mathrm{HD}}\right) \hat{U}_{S}^{A B}\right]\right],
\end{aligned}
$$

where $\hat{U}_{S}^{A B}$ is an $\left(N_{A}+N_{B}\right)$-mode Gaussian unitary operation and $\left\{\hat{\Pi}_{\mathrm{HD}}^{A B}\left(\Gamma_{\mathrm{HD}}, d_{\mathrm{HD}}\right)\right\}$ represents $N_{A}+N_{B}$ homodyne detectors with the measurement outcomes denoted by $d_{\mathrm{HD}}$. Note that homodyne detection is a Gaussian measurement (projection onto an infinitely squeezed states) and thus characterized by the covariance matrix. In Eq. (A.1), $\Gamma_{\mathrm{HD}}$ is a $2\left(N_{A}+N_{B}\right) \times 2\left(N_{A}+N_{B}\right)$ diagonal matrix and

$$
\Gamma_{\mathrm{HD}}=\operatorname{diag}\left[e^{-2 r}, e^{2 r}, e^{-2 r}, e^{2 r}, \cdots, e^{2 r}\right],
$$

with $r \rightarrow \infty$.

Equation (A.1) implies that the POVM of the Gaussian measurement model is derived from a set of operators $\left\{\operatorname{Tr}_{B}\left[\hat{\rho}_{\mathrm{aux}}^{B} \hat{U}_{S}^{A B \dagger} \hat{\Pi}_{\mathrm{HD}}^{A B}\left(\Gamma_{\mathrm{HD}}, d_{\mathrm{HD}}\right) \hat{U}_{S}^{A B}\right]\right\}_{d_{\mathrm{HD}}}$. Let us describe it by covariance matrices and displacements. Denoting the symplectic transformation corresponding to $\hat{U}_{S}^{A B}$ by $S$, the unitary transformation $\hat{U}_{S}^{A B \dagger} \hat{\Pi}_{\mathrm{HD}}^{A B}\left(\Gamma_{\mathrm{HD}}, d_{\mathrm{HD}}\right) \hat{U}_{S}^{A B}$ is described by

$$
\begin{aligned}
\Gamma_{\mathrm{HD}} & \rightarrow S^{T} \Gamma_{\mathrm{HD}} S \equiv \Gamma_{S}, \\
d_{\mathrm{HD}} & \rightarrow S d_{\mathrm{HD}} \equiv d_{S} .
\end{aligned}
$$


Then after tracing out the ancillary system $B$, we find that the above POVM is given by an $N_{A}$-mode Gaussian operator $\hat{\Pi}_{G}(\Gamma, \delta)$ with

$$
\begin{aligned}
\Gamma & =\Gamma_{A}-\Gamma_{C} \frac{1}{\Gamma_{\mathrm{aux}}+\Gamma_{B}} \Gamma_{C}^{T}, \\
\delta & =d_{A}-\Gamma_{C} \frac{1}{\Gamma_{\mathrm{aux}}+\Gamma_{B}} d_{B},
\end{aligned}
$$

where we have denoted

$$
\begin{aligned}
\Gamma_{S} & =\left[\begin{array}{ll}
\Gamma_{A} & \Gamma_{C} \\
\Gamma_{C}^{T} & \Gamma_{B}
\end{array}\right] \\
d_{S} & =\left[\begin{array}{l}
d_{A} \\
d_{B}
\end{array}\right] .
\end{aligned}
$$

[1] C. W. Helstrom, Quantum Detection and Estimation Theory (Academic Press, New York, 1976).

[2] R. S. Kennedy, Research Laboratory of Electronics, MIT, Quarterly Progress Report No. 108, 1973 (unpublished), p. 219.

[3] S. Dolinar, Research Laboratory of Electronics, MIT, Quarterly Progress Report No. 111, 1973 (unpublished), p. 115.

[4] M. Sasaki and O. Hirota, Phys. Rev. A 54, 2728 (1996).

[5] J. M. Geremia, Phys. Rev. A 70, 062303 (2004).

[6] M. Takeoka, M. Sasaki, P. van Loock, and N. Lütkenhaus, Phys. Rev. A 71, 022318 (2005).

[7] M. Takeoka, M. Sasaki, and N. Lütkenhaus, Phys. Rev. Lett. 97, 040502 (2006).

[8] R. L. Cook, P. J. Martin, and J. M. Geremia, Nature 446, 774 (2007).

[9] S. L. Braunstein and P. van Loock, Rev. Mod. Phys. 77, 513 (2005).

[10] Asymptotic theory of quantum statistical inference: selected papers (edited by M. Hayashi, World Scientific Publishing, New York, 1976).

[11] S. D. Bartlett, B. C. Sanders, S. L. Braunstein, and K. Nemoto, Phys. Rev. Lett. 88, 097904 (2002).

[12] J. Eisert, S. Scheel, and M. B. Plenio, Phys. Rev. Lett. 89, 137903 (2002).
[13] J. Fiurasek, ibid, 89, 137904 (2002).

[14] G. Giedke and J. I. Cirac, Phys. Rev. A 66, 032316 (2002).

[15] N. J. Cerf, O. Krüger, P. Navez, R. F. Werner, and M. M. Wolf, Phys. Rev. Lett. 95, 070501 (2005).

[16] A. Jamiołkowski, Rep. Math. Phys. 3, 275 (1972).

[17] A. S. Holevo, Probabilistic and Statistical Aspects of Quantum Theory (North-Holland, Amsterdam, 1982) Chap. 5.

[18] Note that here we mention the dynamical optimization of only 'quantum' operations. Generally, the measurement outcomes at both steps are necessary for the optimal classical post-processing.

[19] V. A. Vilnrotter and E. R. Rodemich, IEEE Trans. Inf. Theory 30, 446 (1984).

[20] I. Bargatin, Phys. Rev. A 72, 022316 (2005).

[21] D. Rosenberg, A E. Lita, A. J. Miller, and S.-W. Nam, Phys. Rev. A 71, 061803(R) (2005).

[22] J. Yoshikawa, T. Hayashi, T. Akiyama, N. Takei, A. Huck, U. L. Andersen, and A. Furusawa, Phys. Rev. A 76, 060301(R) (2007).

[23] C. Wittmann, M. Takeoka, K. N. Cassemiro, M. Sasaki, G. Leuchs, and U. L. Andersen, Submitted (2008). 